ORIGINAL ARTICLE

\title{
Febrile adults presenting to the emergency department: outcomes and markers of serious illness
}

\author{
J C Knott, S-L Tan, A C Street, M Bailey, P Cameron
}

Emerg Med J 2004;21:170-174. doi: 10.1136/emj.2002.001933

See end of article for authors' affiliations

.....................

Correspondence to:

Dr J C Knott, Department of Emergency Medicine, Royal Melbourne Hospital, Grattan Street, Parkville 3150, Australia;

Jonathan.knot@@mh.org.au

Accepted for publication 1 April 2003

\begin{abstract}
Objectives: To determine outcomes and markers of serious illness for febrile patients presenting to an adult emergency department.

Methods: A prospective cohort study of patients presenting to the emergency department with a temperature $\geqslant 38^{\circ} \mathrm{C}$. Medical staff obtained demographic data and risk factor profiles while assessing each febrile patient. All were followed up to determine death, admission to intensive care, length of stay in hospital, or subsequent admission to hospital within 30 days. Univariate and multivariate analysis determined which factors were markers of serious illness.

Results: For febrile adults admitted to hospital 3.0\% died, $6.1 \%$ were admitted to intensive care, median length of stay in hospital was 7.2 days. Independent risk factors were-death: age $(O R=1.04)$, respiratory rate $(O R=1.06)$, white cell count $(O R=1.02)$, cardiac disease $(O R=3.3)$, and jaundice $(O R=21.4)$. Admission to intensive care: respiratory rate $(O R=1.1)$, pulse rate $(O R=1.03)$, and jaundice $(O R=5.1)$. Increased length of hospital stay: age $(p<0.01)$, jaundice $(p<0.01)$, respiratory rate $(p=0.01)$, focal neurological signs $(p=0.01)$, and changed mental state $(p=0.04)$. For febrile adults sent home $7.9 \%$ required admission to hospital within 30 days. Risk factors were respiratory rate $(O R=1.2)$, being female $(O R=5.36)$, malignancy $(O R=15.3)$, and cardiac disease $(O R=19.7)$. Initially having no focus of infection was protective $(O R=0.13)$. No febrile patient sent home from the emergency department died or required admission to intensive care.

Conclusions: Few febrile adults presenting to the emergency department suffer an adverse outcome suggesting effective risk stratification is occurring. The identification of factors associated with adverse events may further improve this process.
\end{abstract}

$\mathrm{R}$ isk stratification of patients with a raised temperature is one of the most difficult tasks facing clinicians in the emergency setting. Fever is a common presenting symptom to the emergency department (ED) and a raised temperature may be attributable to a wide range of clinical disease. ${ }^{1}$ The disease may be short and self limiting or may result in death or serious morbidity including admission to intensive care (ICU) or prolonged hospital stays. Identification of markers of serious illness would allow resources to be directed to those who need them without excessive use of drugs, particularly antibiotics, or admission to hospital.

There is a large body of literature that examines the febrile paediatric population and identifies factors associated with serious illness, such as absolute increase of temperature, raised neutrophil counts, and detection of band forms. ${ }^{23}$ There have also been a number of studies examining specific populations of patients, such as those admitted to hospital, ${ }^{4-8}$ the immune suppressed, ${ }^{9}{ }^{10}$ and geriatric, ${ }^{11}$ to identify factors associated with poor outcomes. However, no study has sought to determine outcomes and identify factors associated with serious morbidity and mortality in a general adult ED population.

The aim of this study was to determine the outcomes of all adult patients presenting to the ED with fever, and to identify independent risk factors for serious morbidity and mortality among this population.

\section{METHODS}

This was a prospective cohort study of patients presenting to the Royal Melbourne Hospital ED with a tympanic temperature of $38^{\circ} \mathrm{C}$ or greater. Royal Melbourne Hospital is a tertiary referral hospital that also provides community care to a large inner city population. There are about 48000 presentations to the ED per annum and 35\% are admitted.

Temperature was measured using a Genius infrared tympanic thermometer. Tympanic measurements have been highly correlated with core temperature measurements. ${ }^{12}{ }^{13}$ Previous studies have used $37.8^{\circ} \mathrm{C}$ as a temperature cut off, which allows for a normal variation in body temperature up to this level. ${ }^{12}$ To allow for the manufacturer's specified measurement error of $\pm 0.2^{\circ} \mathrm{C}$ for the Genius tympanic thermometer ${ }^{13} 38^{\circ} \mathrm{C}$ was chosen for this study. The upper limit was selected to ensure only patients with a definite increase of temperature were included.

A questionnaire was filled out by the treating physician for all eligible patients to record patient demographics (age and sex), vital signs (temperature, pulse and respiratory rate), and possible risk factors that would contribute to a poor outcome in febrile patients. Risk factors were chosen from published literature by a panel of emergency and infectious diseases physicians. ${ }^{14-7} 91114$ These risk factors were: travel in the previous six months to an endemic malarial area, known human immune deficiency virus infection, a donor organ or bone marrow recipient, chemotherapy or radiotherapy in the previous six weeks, splenectomy, any oral or parenteral catabolic corticosteroid use (inhaled corticosteroids excepted), alcoholism (six or more standard drinks per day), intravenous drug misuse in the past fortnight, clinically or histologically verified malignancy, cardiac, respiratory or renal disease that impacts on daily activities, diabetes, current antibiotic use, rigors, clinical jaundice, vomiting,

Abbreviations: $E D$, emergency department; ICU, intensive care unit 
any rash except those able to be easily identified (for example, shingles), a change from the usual mental state, and new focal neurological signs. Visual analogue scales were used to record severity of headache, deep muscle pain, and pain in other sites with the site noted. The treating doctor was asked to indicate whether at the end of their assessment a focal cause for the inceased temperature could be found. All risk factor definitions were provided in the ED and accessible at all times. A pilot study conducted over two weeks allowed minor modification of the questionnaire to improve compliance. No confusion regarding the definitions arose after the pilot study.

The outcomes assessed for patients admitted to hospital included length of stay in hospital (days), need for admission to ICU or death. The outcomes for those sent home included death, admission to ICU, and hospital admission within the 30 day follow up period.

For admitted patients, including those transferred to other facilities, outcomes were assessed from the clinical record. Discharge diagnoses were also obtained. All patients sent home were followed up at a minimum of 30 days to determine subsequent need for hospital or ICU admission or death. The principal investigator made up to three telephone calls to obtain this information and then sent two written requests to the last known address if telephone contact was unsuccessful. If no contact was made the hospital computer records were searched for a later attendance to ED or an admission.

From the computer records for each presentation, total white cell and neutrophil counts were determined, if ordered, as well as the results of any blood cultures taken within 24 hours of presentation to the ED.

The questionnaires were complete except for eight patients with missing respiratory rates. These were obtained from the initial nursing notes recorded in the ED.

\section{Statistical analysis}

All data were analysed using Stata version 6 and SAS version 8.0. Age, temperature, and respiratory rate were recorded as continuous variables. Total white cell and neutrophil counts, as well as the outcome length of stay were also continuous, but required log transformation to produce a normal distribution. All other variables were categorical. The presence or absence of headache, deep muscle pain, or other pain discriminated between outcomes but no further information was obtained from the degree of pain recorded so these variables were also treated as categorical data.

Univariate analysis was conducted using $\chi^{2}$ tests for equal proportion, Fisher's exact tests, Pearson's correlation, coefficients, Student's $t$ test, and analysis of variance where appropriate.

Multivariate analysis was conducted using multiple linear regression for continuous outcomes and multiple logistic regression for categorical outcomes. Multivariate models were constructed using a stepwise selection procedure and then validated using a backward elimination procedure. Significance was set at $\mathrm{p}<0.05$.

\section{Sample size}

Because no previous study has sought to determine outcomes and identify factors associated with serious morbidity and mortality in a general adult ED population, this study was exploratory in nature. Based on historical data, it was anticipated that during a 12 month period, about 800 subjects would present to the ED with raised temperatures. Assuming a case/control ratio of $1 / 20$, this study had an $80 \%$ power to detect a difference in normally distributed outcomes equivalent to 0.5 of a standard deviation with a two sided $p$ value of 0.05 . Similarly, this study has an $80 \%$ power to detect a $25 \%$ difference in proportion with a two sided p value of 0.05 .

\section{RESULTS}

\section{Sample description}

Over 12 months, 803 patients were enrolled from 837 presentations, consistent with the predicted total of 730 (based on pilot data). Of these, 609 (72.8\% of the total, 95\% CI: $69.8 \%$ to $75.7 \%$ ) were admitted to hospital consistent with the pilot admission rate of $75 \%$. Thirty seven patients $(6.1 \%$ of those admitted, $95 \%$ CI: $4.2 \%$ to $7.9 \%$ ) went to ICU and 22 (3.6\%, 95\% CI: $2.2 \%$ to $5.0 \%$ ) died. Of the 228 patients sent home, 18 patients $(7.9 \%, 95 \%$ CI: $4.4 \%$ to $11.4 \%)$ were admitted into hospital within 30 days of their initial presentation to the ED and 32 (14\%, 95\%CI: $9.5 \%$ to $18.5 \%$ ) were lost to follow up. No patient sent home died (95\% CI: $0 \%$ to $1.7 \%, \mathrm{p}<0.001$ ) or subsequently required ICU (95\% CI: $0 \%$ to $1.7 \%, \mathrm{p}=0.006)$. The mean age of all patients was 49 $(\mathrm{SD}=22)$. For those admitted to hospital, there was no significant difference between the mean age of all patients ( 54 years, $\mathrm{SD}=22$ ), those sent to ICU ( 55 years, $\mathrm{SD}=20$ ), or those that died ( 68 years, $S D=18$ ). There was no significant difference between the mean ages for all patients sent home ( 36 years, $S D=17$ ), those readmitted ( 42 years, $S D=20$ ), and those lost to follow up ( 34 years, $S D=17$ ). Fifty seven per cent of all patients were male and there was no significant gender difference between the categories. In particular, $58 \%$ of admitted patients were male and $53 \%$ of those sent home $(\mathrm{p}=0.183)$. The median temperature of all patients was $38.7^{\circ}$ (interquartile range $(\mathrm{IQR})=38.2-39.1$ ); this median varied up to $0.2^{\circ}$ between the other categories (a non-significant difference). The median length of stay in hospital was 7.2 days (IQR $=3-8)$ compared with the median for all non-same day medical patients at our hospital of 5.6 days. A white cell count and differential was available for $95.1 \%$ of patients, $99.5 \%$ of those admitted, and $83.7 \%$ of those sent home. Blood was sent for culture in $88 \%$ of all patients, $90.5 \%$ of admitted patients, and $64.9 \%$ of patients sent home. Table 1 shows the most frequent hospital

\begin{tabular}{lll} 
Table 1 Discharge diagnosis of febrile patients \\
\hline Category & Number & $\%$ Of total \\
\hline Infectious & 367 & 61 \\
Pneumonia & 119 & 19.7 \\
Septicaemia & 53 & 8.8 \\
Inf Exac COAD & 30 & 5.0 \\
Cellulitis & 23 & 3.8 \\
Fever for investigation & 22 & 3.6 \\
UTI & 20 & 3.3 \\
URTI & 19 & 3.1 \\
Meningitis & 16 & 2.6 \\
Malaria & 5 & 0.8 \\
Surgical & 85 & 14 \\
Cholecystitis & 16 & 2.6 \\
Appendicitis & 11 & 1.8 \\
Postoperative complication & 8 & 1.3 \\
Diverticulitis & 7 & 1.2 \\
Peritonitis & 2 & 0.3 \\
Pancreatitis & 2 & 0.3 \\
Other & 152 & 25 \\
Nephritis & 26 & 4.3 \\
Fractures & 10 & 1.7 \\
Pneumonitis & 8 & 1.3 \\
Arthritis & 8 & 1.3 \\
Malignancy & 7 & 1.2 \\
Epilepsy & 7 & 1.2 \\
\hline
\end{tabular}

Final discharge diagnosis from medical records. Five patients unavailable because of hospital transfer. N, number of patients; Inf Exac COAD, infective exacerbation of chronic obstructive airways disease; URTI, upper respiratory tract infection; UTI, urinary tract infection. 
discharge diagnosis for admitted patients. This was unavailable for five patients transferred to other hospitals.

\section{Factors associated with death, ICU admission, and length of stay}

For admitted patients several factors were significantly associated with death (table 2), ICU admission (table 3), or length of stay (table 4). After multivariate analysis those factors that continued to be significant for death were age, respiratory rate, total white cell count, significant cardiac disease, and jaundice. For admission to ICU independent risk factors were respiratory rate, pulse rate, and jaundice. While for length of stay in hospital independent risk factors were age, respiratory rate, jaundice, changed mental state, and focal neurological signs. As length of stay in hospital was log transformed for analysis, odds ratios are unavailable so $\mathrm{p}$ values are presented, together with the parameter estimates and standard deviations.

\section{Factors associated with admission to hospital at follow up}

Eighteen patients reported that they had been admitted to hospital within 30 days of their initial presentation to the ED. These patients were likely to be female or had an increased respiratory rate, malignancy, cardiac disease, or initially had no focus of infection (table 5).

There was successful follow up of $86 \%$ of patients sent home. There was no significant difference, for any preidentified risk factor, between the patients successfully followed up and the 32 patients that were unable to be contacted. Half of those lost were overseas visitors that did not respond to written follow up and had no available phone contact. The remainder had either incorrect details or appeared to be itinerant.

\section{DISCUSSION}

This study identified the outcomes of all patients with a fever that presented to an adult, tertiary referral, ED, including patients admitted and those discharged home. In addition, risk factors associated with important adverse outcomes were determined. This is the first study to look at the entire presenting population and not select groups. Selection bias appears to have been minimal given the close approximation of patient numbers and disposition with those predicted by the pilot study and the high rate of successful follow up.

Risk factors associated with death, admission to ICU, and length of stay for admitted patients

Respiratory rate and jaundice were the only factors, after logistic regression, to be significant for all three adverse events for admitted patients. While respiratory rate has previously been shown to be associated with a poor outcome, ${ }^{11}$ there are no published studies of febrile ED patients that have shown an association with jaundice and adverse outcomes. However, fever and jaundice are hallmarks of ascending cholangitis and septicaemia with inherently high mortality. ${ }^{15} 16$

Age $>50$ years old has been used to stratify groups of febrile patients at risk ${ }^{5-8}$ but in our study age was kept as a continuous variable to determine the increasing odds of adverse outcomes. While increasing age was associated with death and increased length of stay in hospital, the lack of association with admission to ICU may be attributable to a selective admission policy to this unit.

Leucocytosis is cited in the paediatric literature as a marker of occult bacteraemia ${ }^{23}$ and raised white cell counts have been associated with adverse outcomes in general and geriatric populations..$^{5-811}$ In our study the risk of death increased progressively with the white cell count.

The association of new focal and general neurological abnormality with length of stay in hospital may be attributable to the increased difficulty in discharging patients home with these deficits.

\section{Risk factors associated with subsequent admission to hospital within 30 days}

Known malignancy, increased respiratory rate and cardiac disease were associated with admission within 30 days of the index presentation to the ED.

The reason for women having a 5.4-fold subsequent admission rate is not clear but may relate to a propensity to return to hospital if not improving. However, the defined outcome is admission to hospital and not just reassessment in the ED. There was no gender difference for initial admission to hospital.

Having no focus of infection when seen in the ED was protective and decreased subsequent admission nearly eightfold. This is consistent with the younger population, with generally fewer risk factors, that was discharged from the ED and in which no focus of infection may correspond to systemic viral illnesses.

Of the patients sent home from the ED, $7.9 \%$ required admission to hospital within 30 days. It was assumed that all subsequent admissions were atributable to the original febrile illness but as these patients included, for example, oncology patients receiving courses of chemotherapy it is probable that at least some of these representations were unrelated to the index presentation. However, admission was the defined outcome and it was deemed too subjective to identify which patients had developed a second, independent illness.

Table 2 Risk factors associated with death of admitted patients

\begin{tabular}{|c|c|c|c|c|c|c|}
\hline \multirow[b]{2}{*}{ Variable } & \multicolumn{2}{|l|}{ Incidence } & \multirow[b]{2}{*}{$\begin{array}{l}\text { Unadjusted } \\
\mathrm{p} \text { value }\end{array}$} & \multirow[b]{2}{*}{$\begin{array}{l}\text { Adjusted } \\
\text { p value }\end{array}$} & \multirow[b]{2}{*}{ Adjusted OR } & \multirow[b]{2}{*}{$95 \% \mathrm{Cl}$} \\
\hline & $\begin{array}{l}\text { Deaths } \\
n=22(\%)\end{array}$ & $\begin{array}{l}\text { No death } \\
n=587(\%)\end{array}$ & & & & \\
\hline Age $^{*}$ & $68(18) \dagger$ & $54(22) \dagger$ & 0.01 & 0.02 & 1.04 & 1.01 to 1.07 \\
\hline $\mathrm{RR}^{*}$ & $24(6.8) \dagger$ & $20(5.6) \dagger$ & 0.01 & 0.03 & 1.06 & 1.01 to 1.12 \\
\hline WCC* & $9.9(11.3) \dagger$ & $11.3(8.5) \dagger$ & 0.80 & 0.02 & 1.02 & 1.00 to 1.03 \\
\hline Cardiac & $8(36)$ & $80(14)$ & $<0.01$ & 0.03 & 3.28 & 1.14 to 9.45 \\
\hline Jaundice & $3(14)$ & $12(2)$ & $<0.01$ & $<0.01$ & 21.46 & 4.83 to 95.4 \\
\hline Pos BC & 7 (32) & 72 (12) & 0.01 & 0.44 & NA & \\
\hline Malignancy & 7 (32) & 71 (12) & 0.01 & 0.07 & NA & \\
\hline Diabetes & $6(27)$ & 74 (13) & 0.05 & 0.77 & NA & \\
\hline Headache & $1(5)$ & $156(26)$ & 0.02 & 0.91 & NA & \\
\hline
\end{tabular}

Incidence, number of patients for which this variable occurred. Unadjusted $p$ value, univariate analysis. Adjusted $p$ value, $\mathrm{p}$ value adjusted for all other variables. ${ }^{*}$ Continuous variable so that the $O R$ increases incrementally with variable; †median (SD); RR, respiratory rate; WCC, total white cell count; Pos BC, positive blood culture within 24 hours of presentation; NA, not applicable. 
Table 3 Risk factors associated with admission to ICU

\begin{tabular}{|c|c|c|c|c|c|c|}
\hline \multirow[b]{2}{*}{ Variable } & \multicolumn{2}{|l|}{ Incidence } & \multirow[b]{2}{*}{$\begin{array}{l}\text { Unadjusted } \\
\text { p value }\end{array}$} & \multirow[b]{2}{*}{$\begin{array}{l}\text { Adjusted } \\
\text { p value }\end{array}$} & \multirow[b]{2}{*}{ Adjusted OR } & \multirow[b]{2}{*}{$95 \% \mathrm{Cl}$} \\
\hline & $\begin{array}{l}\text { ICU } \\
n=37(\%)\end{array}$ & $\begin{array}{l}\text { No ICU } \\
n=572(\%)\end{array}$ & & & & \\
\hline Pulse* & $110(30) t$ & $100(18) \dagger$ & 0.02 & $<0.01$ & 1.03 & 1.01 to 1.04 \\
\hline $\mathrm{RR}^{*}$ & $24(8.5) \dagger$ & $20(5.4) \dagger$ & $<0.01$ & $<0.01$ & 1.10 & 1.05 to 1.14 \\
\hline Jaundice & $3(8)$ & $12(2)$ & 0.02 & 0.02 & 5.12 & 1.29 to 20.4 \\
\hline Splenectomy & $2(5)$ & $3(0.6)$ & $<0.01$ & 0.17 & NA & \\
\hline AMS & $10(27)$ & 74 (13) & 0.02 & 0.21 & NA & \\
\hline
\end{tabular}

A number of limitations in this study have been identified. The selection and definition of risk factors required a consensus approach by a panel of emergency and infectious diseases physicians because of either a lack of accepted agreement on terms or multiple definitions in the literature. Risk factors and their definitions were made available to ED medical staff for the duration of the study to maintain uniformity.

This study looked at the febrile population as an entity. A raised temperature may have been attributable to a minor comorbidity such as a urinary tract infection in a patient with an acute cerebrovascular accident. Conversely, an increased temperature may add comparatively little clinical information to aid the diagnosis of fulminant peritonitis. However, as there has been no published literature about this group of patients this initial study does not attempt to discriminate on the relative value of the fever to the emergency physician.

There are several other outcomes that could have been assessed including bacteraemia rates and empirical need for antibiotics among the cohort. These outcomes assume an infective cause for the raised temperature or require a subjective judgement by the clinicians, for example, need to give antibiotics. Conversely, the outcomes for this study are applicable to the whole population and can be assessed objectively.

Of the patients sent home, 32 (14\%) were lost to follow up. A few adverse outcomes among this group would significantly affect our results. However, the lost to follow up patients tended to be young (mean age 34) and with few or no risk factors for serious illness. They had a similar demographic profile to those that could be followed up and had no significant difference for any of the risk factors. Thus, it is reasonable to assume that there was not an increased adverse event rate among this group.

Finally, this study had four outcomes, two separate groups of patients (admitted and sent home) and 26 risk factors that were assessed. It is expected that significant associations would occur by chance. In addition co-linearity is likely to exist between some of the variables resulting in confounding of the results. These problems were dealt with by stepwise logistic regression and excluding those risk factors that lost significance (tables 2-5).

In conclusion, this study is the first to assess the outcomes of all febrile adult patients presenting to an adult ED. The overall mortality and serious morbidity rates are low. For patients sent home from the ED the outcomes are excellent with no subsequent deaths or admission to ICU within 30 days. This most probably shows that the ED staff were able to clinically stratify febrile patients into a low risk group and it is very unlikely that this would have happened by chance (probability of 0 deaths $=0.006$ and probability of 0 ICU admissions $<0.001$ respectively).

Independent risk factors were also identified for important adverse outcomes. These factors, readily assessable in the ED, are able to provide assistance when assessing febrile patients within the ED and stratifying those who are at risk of a poor outcome. Further research needs to be done to identify outcomes and risk factors in selected subgroups, including those for which no cause for the fever could be found in the

\begin{tabular}{|c|c|c|c|c|c|}
\hline Variable & $\begin{array}{l}\text { Incidence } \\
n=609(\%)\end{array}$ & $\begin{array}{l}\text { Unadjusted } \\
\text { p value }\end{array}$ & $\begin{array}{l}\text { Adjusted } \\
\mathrm{p} \text { value }\end{array}$ & $\begin{array}{l}\text { Parameter } \\
\text { estimate }\end{array}$ & Standard error \\
\hline Jaundice & $15(2.5)$ & $<0.01$ & $<0.01$ & 0.79 & 0.20 \\
\hline Age† & $54(22) \ddagger$ & $0.36 \S$ & $<0.01$ & 0.01 & $<0.01$ \\
\hline RR† & $20(5.7) \ddagger$ & $0.23 \S$ & 0.01 & 0.02 & 0.01 \\
\hline Focal neuro & $18(3)$ & $<0.01$ & 0.01 & 0.47 & 0.19 \\
\hline CMS & 84 (14) & $<0.01$ & 0.04 & 0.20 & 0.10 \\
\hline Diabetes & 80 (13) & $<0.01$ & 0.08 & & \\
\hline Headache & $157(26)$ & $<0.01$ & 0.12 & & \\
\hline Travel & $30(4.9)$ & 0.01 & 0.43 & & \\
\hline Rigors & $177(29.1)$ & 0.01 & 0.51 & & \\
\hline Muscle pain & 115 (18.9) & 0.01 & 0.60 & & \\
\hline Cardiac & $89(14.6)$ & 0.02 & 0.32 & & \\
\hline Pos BC & 79 (13) & 0.03 & 0.83 & & \\
\hline Vomiting & $158(26)$ & 0.04 & 0.85 & & \\
\hline Malignancy & 78 (13) & 0.04 & 0.10 & & \\
\hline No focus found & $423(70)$ & 0.04 & 0.92 & & \\
\hline \multicolumn{6}{|c|}{$\begin{array}{l}\text { *Length of stay was log transformed for analysis. Incidence, number of patients for which this variable occurred. } \\
\text { Unadjusted } p \text { value, univariate analysis. Adjusted } p \text { value, } p \text { value adjusted for all other variables. TContinuous } \\
\text { variable so that the OR increases incrementally with each unit increase in variable; †median (SD); scorrelation } \\
\text { between continuous variables; Focal neuro, focal neurological signs; Travel, travel to an endemic malarial area; } \\
\text { Cardiac, cardiac disease affecting daily activity; Pos BC, positive blood cultures. Other abbreviations as in table } 3 .\end{array}$} \\
\hline
\end{tabular}


Table 5 Variables associated with subsequent admission of febrile patients discharged from the ED

\begin{tabular}{lcccccc}
\hline & \multicolumn{2}{l}{ Incidence } & & & & \\
\cline { 2 - 4 } Variable & $\begin{array}{l}\text { Admission } \\
\mathbf{n = 1 8}(\%)\end{array}$ & $\begin{array}{l}\text { No admit } \\
\mathbf{n = 2 1 0}(\%)\end{array}$ & $\begin{array}{l}\text { Unadjusted } \\
\text { p value }\end{array}$ & $\begin{array}{l}\text { Adjusted } \\
\mathbf{p} \text { value }\end{array}$ & Adjusted OR $\mathbf{9 5 \% ~ C l}$ \\
\hline No focus found & $5(28)$ & $70(33)$ & 0.01 & 0.01 & 0.13 & 0.03 to 0.55 \\
Female & $11(61)$ & $80(38)$ & 0.19 & 0.02 & 5.36 & 1.36 to 21.2 \\
Malignancy & $3(17)$ & $3(1.5)$ & $<0.01$ & 0.02 & 15.32 & 1.55 to 151.9 \\
RR* & $17(5.0) \dagger$ & $18(6.6) \dagger$ & 0.16 & 0.03 & 1.16 & 1.01 to 1.33 \\
Cardiac & $1(6)$ & $3(1.5)$ & 0.28 & 0.04 & 19.71 & 1.12 to 345.9 \\
Chemotherapy & $2(11)$ & $1(0.5)$ & $<0.01$ & 0.06 & NA & \\
Corticosteroids & $2(11)$ & $4(2)$ & 0.04 & 0.20 & NA & \\
IVDU & $1(6)$ & $1(0.5)$ & 0.05 & 0.05 & NA & \\
\hline
\end{tabular}

Incidence, number of patients for which this variable occurred. Unadjusted $p$ value, univariate analysis. Adjusted $p$ value, $\mathrm{p}$ value adjusted for all other variables. ${ }^{*}$ Continuous variable so that the OR increases incrementally with each unit increase in variable; tmedian (SD); Corticosteroids, use of non-inhaled catabolic corticosteroids; IVDU, intravenous drug user. Other abbreviations as in tables 3 and 4 .

ED. Research should also be undertaken to determine whether incorporation of these risk factors in patient stratification improves outcomes or could be used to change disposition from the ED.

\section{Authors' affiliations}

J C Knott, S-L Tan, Department of Emergency Medicine, Royal Melbourne Hospital, Melbourne, Australia

A C Street, Victorian Infectious Diseases Service, Royal Melbourne Hospital

M Bailey, Department of Mathematical Sciences, Swinburne University, Hawthorn, Australia

P Cameron, Accident and Emergency Academic Unit, The Chinese University of Hong Kong, Prince of Wales Hospital, Shanin, NT, Hong Kong

Funding: there was no independent funding for this study outside of the support provided by the host institution, including salaries and infrastructure.

Conflicts of interest: none.

This study was submitted to and approved by the Royal Melbourne Hospital Ethics Committee and conforms to the principles of the Declaration of Helsinki. This study contains original work and has not been published in part or in total elsewhere.

\section{REFERENCES}

1 Yung A. Approach to undifferentiated fever in adults. In: Cameron $\mathrm{P}$, Jelinek G, Kelly A, et al, eds. Textbook of adult emergency medicine. 1 st edn. Edinburgh: Churchill Livingston, 2000:303-7.
2 Baraff LJ, Bass JW, Fleisher GR, et al. Practice guideline for the management of infants and children 0 to 36 months of age with fever without source. Ann Emerg Med 1993;22:108-19.

3 Haddon RA, Barnett PA, Grimwood K, et al. Bacteraemia in febrile children presenting to a paediatric emergency department. Med J Aust 1999;170:475-8.

4 Manning LV, Touquet R. The relevance of pyrexia in adults as a presenting symptom in the accident and emergency department. Arch Emerg Med 1988;5:86-90.

5 Mellors JW, Horowitz RI, Harvey MR, et al. A simple index to identify occult bacterial infection in adults with acute unexplained fever. Arch Intern Med 1987; 147:666-71.

6 Leibovici L, Greenshtain S, Cohen O, et al. Bacteraemia in febrile patients. Arch Intern Med 1991;151:1801-6.

7 Leibovici L, Cohen O, Wysenbeek AJ. Occult bacterial infection in adults with unexplained fever. Arch Intern Med 1990;150:1270-2.

8 Gallagher JE, Brooks F, Gennis P. Identification of serious illness in febrile adults. Am J Emerg Med 1994;12:129-33.

9 Marantz $\mathbf{P}$, Linzer $M$, Feiner CJ. Inability to predict diagnosis in febrile intravenous drug abusers. Ann Intern Med 1987;106:823-8.

10 Wrenn KD, Larson S. The febrile alcoholic in the emergency department. Am J Emerg Med 1991;9:57-60.

11 Marco CA, Schoenfeld CN, Hansen KN, et al. Fever in geriatric emergency patients: clinical features associated with serious illness. Ann Emerg Med 1995; $26: 18-24$

12 Stewart JV, Webster D. Re-evaluation of the tympanic thermometer in the emergency department. Ann Emerg Med 1992;21:158-61.

13 McKenzie N, O'Hara G, Bayham E, et al. Clinical considerations for use of FirstTemp and Genius infrared tympanic thermometers. St Louis: Sherwood Medical, 1996.

14 Kelly A. Clinical impact of blood cultures taken in the emergency department. $J$ Accid Emerg 1998;15:254-6.

15 Rodgers I, Dunn R. Biliary disease. In: Dunn R, ed. The emergency medicine manual. 2nd edn. Adelaide: Venom Publishing, 2000:176.

16 Jui J. Septic shock. In: Tintinalli J, Kelen GD, Strapczynski JS, eds. Emergency medicine: a comprehensive study guide. 5th edn. New York: McGraw-Hill, 1999:229-38. 